\title{
THE RELATIONSHIP BETWEEN INCOME AND HEALTH. COMPARATIVE STUDY ROMANIA VS. UKRAINE
}

\author{
Ramona Marinela Simuț*, Diana Claudia Perțicaș \\ Department of Economics, Faculty of Economic Sciences, University of Oradea, Oradea, \\ Romania \\ simut.ramona@yahoo.com \\ dianaperticas@yahoo.com
}

\begin{abstract}
The hereby work plans on analysing to what extent the life expectancy is subject to the influence by different markers, like: GDP, the percentage of GDP allocated for the health system, as well as the income level in Romania and Ukraine. We consider that a comparative analysis between the two countries is representative since, as it results from the data and the diagrams presented within the work, both the GDP evolution and the percentage allocated from GDP for the health system reveal a different evolution, ranging in both countries, unlike the life expectancy going through an ascending trend, slow for both cases. Partially considering the specialized literature in use, our attention was drawn towards the evolution of the income obtained by the people. More precisely, commencing from Keynes' Basic Psychological Law stipulating that along with the income growth the expenditure on health, education and training the human resource will increase, we have chosen to econometrically test these theories for Romania and Ukraine. Therefore, we would like to research, using a simple linear regression model, whether the income growth, which represents the independent variable, obtained by the people, has a significant contribution in life expectancy (the dependent variable) increase in Romania, as well as in Ukraine.
\end{abstract}

Keywords: life expectancy; GDP; individual income; wage.

JEL classification: C12; I12.

\section{The connection between income and life expectancy of the population}

The connection between the costs-income levels but especially consumption, was greatly covered during the years. The most popular writing in this sense is The Fundamental Psychological Law formulated by the renowned English economist John M. Keynes, according to which "as a general rule, and in average, people tend to increase consumption when income is higher, but not by how much the income has risen" (Florea, 2008: 64).

Analyzing this we must not overlook the fact that in Romania, most part of individuals spend their personal gains on assuring the bare necessities as Maslow's pyramid shows. Supply of energy and home consumption warm water is one of the primary needs of economic agents, which is a significant percentage from the basic costs of the population.

About the variations of demand based on income F. Engel, economist and statistician, wrote a series of observations currently found in the literature under the name of "Engel's laws", as follows: according to the first law, the greater the income, the greater the provision costs but only in absolute value, because in relative value there is a decrease recorded. In the second and third laws, Engel considers that the share of expenses for clothing, fuel, household and illumination will stay the same irrespective of the income share only to state in his fourth law

\footnotetext{
${ }^{*}$ Corresponding author
} 
that the sundry expenses will shift depending on the fluctuations registered by income. These laws came to life after various economical-statistical investigations but the present conclusion is that only the first and last of the laws are working in the existing economic context.

Also supported by economic investigations, the economists Pranab Bardhan and Christopher Udry (Bardhan and Udry, 2009) have identified a series of economical-social implications resulted after the alteration of the available income in a positive sense. They have conducted various studies and investigations both in developed countries and in developing ones concluding that in the developed countries the income increase would determine increase of expenses with health, education and updating human capital, as well as with raising and education of children. In the developing countries, costs go to health and the acquisition of high technology goods. For the latter, it was concluded that there is a reverse relation between the available income and peoples' desire to emigrate. From the category of negative effects of economic growths, the most frequent is the decrease of the rate of reproduction both in developing countries and especially in the developed ones.

Another no less interesting angle is the one presented by the economists Costea Carmen, Popescu Constantin and Taşnadi Alexandru, who stated that there is a direct proportional connection between the income level and the number of healthy days. In other words, "a higher wages means a greater stock of health" (Costea, Popescu and Tașnadi, 2010: 350).

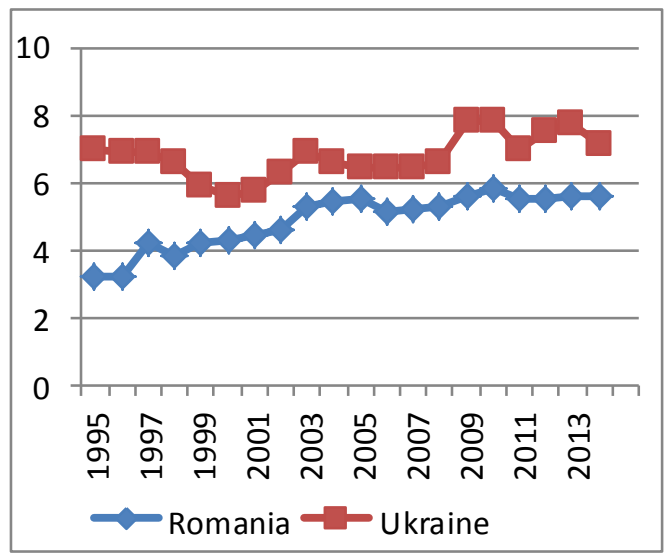

Figure 1: Total expenditure on health $\%$ of GDP in Romania vs. Ukraine Source: Figures drawn up by the authors based http://apps.who.int/nha/database/Key In-dicators by Country/Index/en, accessed on the $11^{\text {th }}$ of July, 2016

In the above presented figures, it is obvious that, in both cases studied, life expectancy was on a rising trend. Now we are well aware that this owes to the new discoveries made in medicine, techniques, technology, diet, which, at least in the analyzed countries, is plentiful. As we speak more and more about how our present way of life is not quite a healthy one, that our diet contains many substances bad for us, we wished to see if hope for a healthy life has increased at the same time with life expectancy in general (Badulescu and Badulescu, 2014). This factor indicates "average life duration spent in different conditions: total survival, survival without chronic diseases and handicap free survival" (European Health \& Life Expectancy).

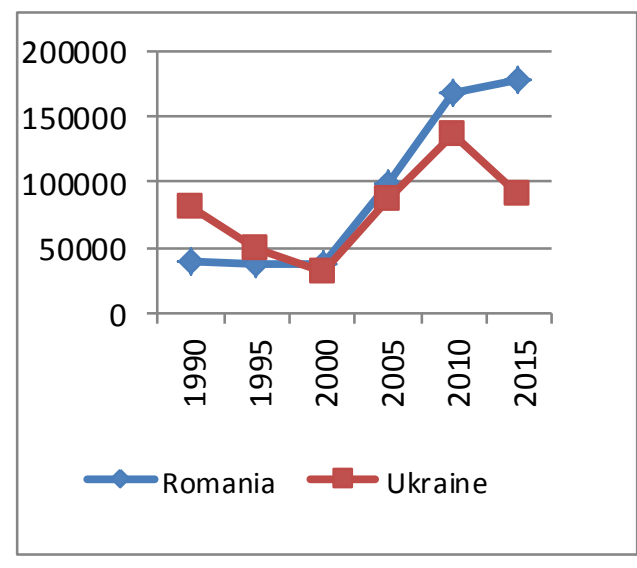

Figure 2: GDP at market prices (current as US\$-billion) in Romania vs. Ukraine on the data published on the World Health available online at (n) 
What is peculiar is that Ukraine, though it settles a larger percentage of the GDP for the health system, scores a lesser life expectancy than Romania and even an inferior ,healthy life expectancy" than Romania, as it will be noticeable in the next figures. It is beyond doubt that the size of GDP is what really matters, therefore we directed our attention to its evolution also. Considering the figure 4 presenting the GDP evolution in both analyzed countries, we may ascertain that it went through major changes compared to the life expectancy or the healthy life expectancy, where the trend is ascension and almost linear.



Figure 3: Life expectancy at birth in Romania vs. Ukraine

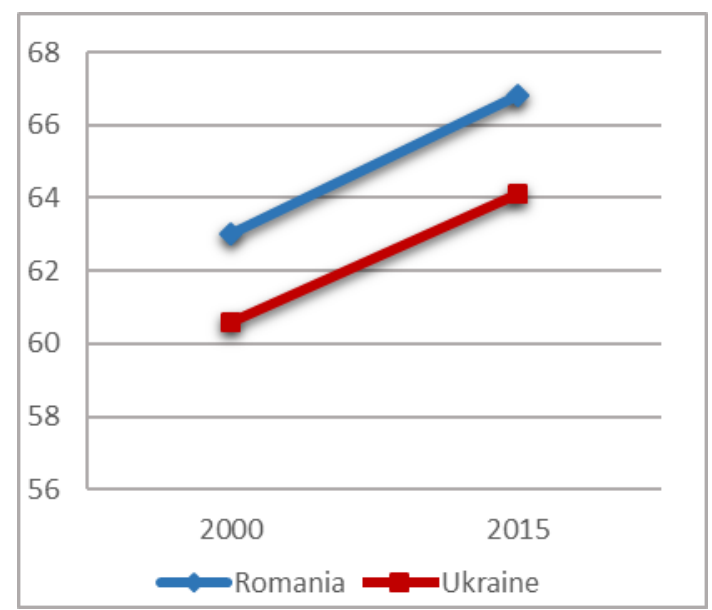

Figure 4: Healthy life expectancy at birth in Romania vs. Ukraine

Source: Figures drawn up by the authors based on the data published on the World Health Organization website available online at http://apps.who.int/nha/database/Key In-dicators by Country/Index/en, accessed on the $11^{\text {th }}$ of July, 2016

As it may be concluded from the above figures, individuals not only that they live longer, but they also have a higher healthy life expectancy. Nevertheless, the size of GDP as well as the percentage allocated from it towards the health system has a significant contribution on the life expectancy increase; we may notice that they directly reflect on each other, though at the first glance it is not extremely powerful.

After analysing part of the macroeconomic factors contributing to the increase of life expectancy, we will approach to the microeconomic factors, respectively we would like to analyse the size of the individual income contributing to the life expectancy increase. Further on, we would like to find out if to the increase of these factors also contribute, in a significant way, the increase of wages obtained by the individuals, fact that allow them to pay greater attention on the quality of life.

\section{Empirical evaluation of the relationship between life expectancy and income}

In analysing simple regression, firstly we take into account aspects related to identifying explanatory variables. We will use data referring to the life expectancy $\left(\operatorname{LE}_{t}\right)$ and the income from Romania and Ukraine, during 1990 - 2014. Starting from Milton Friedman's theory according to which the consumption is directly proportional to the permanent income, in this model we considered that the permanent income of an individual is the salary received by him. The difference between total and permanent income (wages in this case) is called transitory income by Friedman. It should also be specified and that income includes amount of money obtained from various sources such as gifts, bonuses etc., thus the real value of 
the income of an individual is not known exactly, which is why in our analysis the income will be measured with the help of the wages indicator $(W)$, this indicator being part of the income.

Starting from these variables, we want to identify the impact the wage has on the life expectancy. Given the exponential evolution of the variables considered, the numerical values were transformed by logarithm and the influence of seasonal factors was eliminated using Tramo-Seats method. The new variables were further symbolized in case of Romania: life expectancy - Log_LER and the wage - Log_WGR, while in the case of Ukraine: life expectancy - Log_LEU and the wage - Log_WGU.

Specifying an econometric model assumes choosing a mathematical function $(f(x))$ with the help of which the connection between the two variables can be described. In the case of a single factor model, the most common used method is the graphical representation of the two strings of values with the help of the scatter plot. Thus, in order to identify the relation between the mentioned variables, we have created a graphic representation of the pair of points which include the values of the variables, the life expectancy and the wages (in figure 5 we have the Romanian case and in the figure 6 we have the Ukraine case). Based on the data provided by World Bank, The National Institute of Statistics of Romania (INSSE), The State Statistics Service of Ukraine and using the Eviews software, we have obtained the following results:
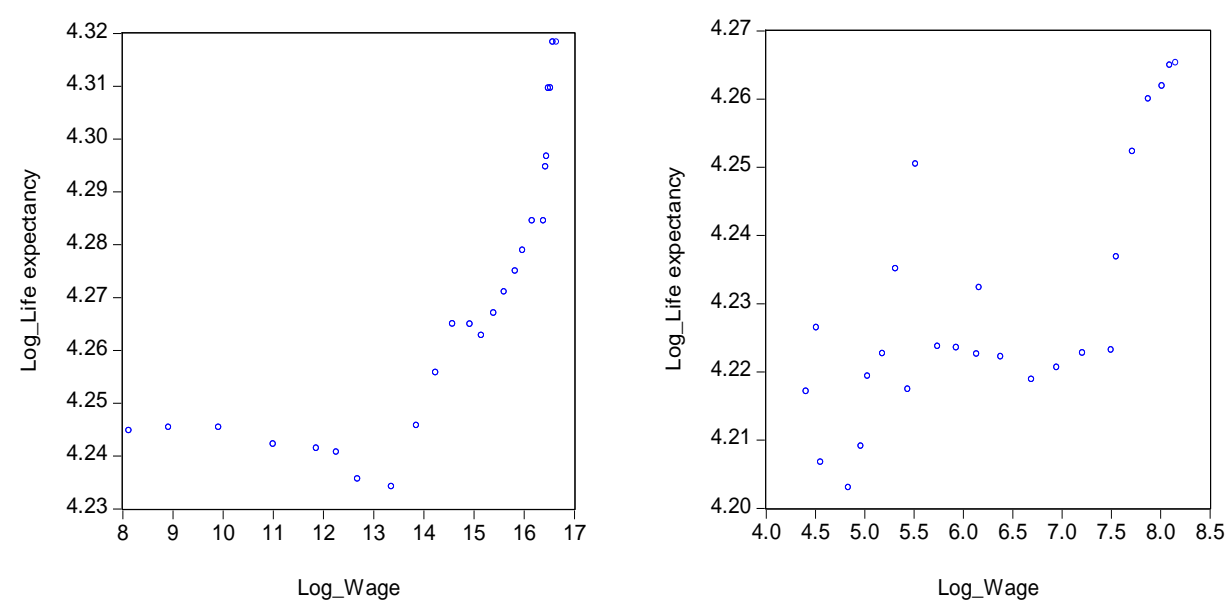

Figure 5: LER-WGR correlation in Romania Figure 6: LEU-WGU correlation in Ukraine Source: authors' representation using Eviews software based on the data provided by World Bank, INSSE and The State Statistics Service of Ukraine

Seeing that the points are situated in a well-defined area in both figures, we can say that between the dependent variable - life expectancy and the independent variable -wage there is a direct connection. In order to identify the connection between these variables and of the intensity of their connection, we will suppose that between the variables there is a linear connection form.

\subsection{The correlation between life expectancy and wage in Romania}

The simple linear regression model proposed in studying the evolution of the life expectancy $(\mathrm{Y})$ in relation to the wages $(\mathrm{X})$ in Romania has the form:

$$
\text { Log_LER }=c(1)^{\star} \text { Log_WGR+c(2)+ } \varepsilon_{t}
$$


The main problem of each regression model is determining the parameters of the model. For determining these parameters, we will use the generalized least squares method. In order to test the validity of the hypothesis on which the classic model is based on, various statistical tests will be used. To estimate the parameters of the model (1), we have used the Eviews program, with the help of which we obtained the following results at a sample level:

Table 1: The estimation of regression equation (1)

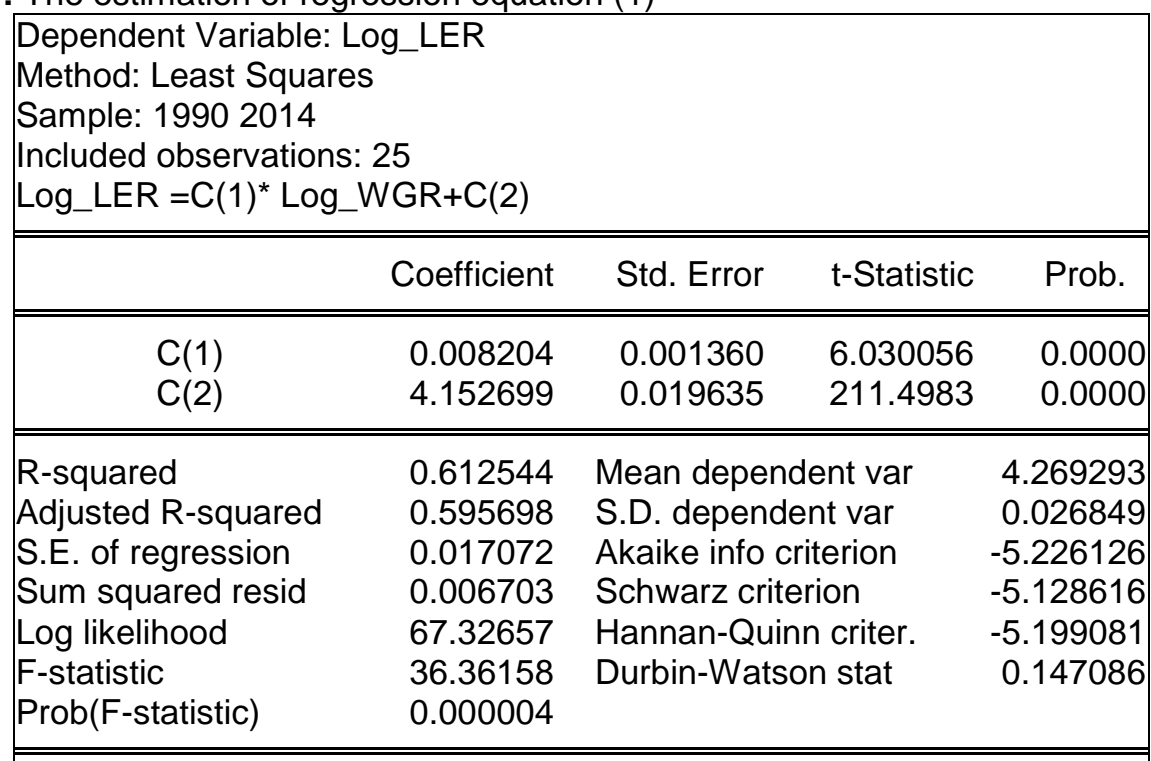

Source: authors' estimates using Eviews based on the data provided by World Bank and INSSE

According to the table above, we can notice the existence of a direct relation between wages and life expectancy. Therefore, for an increase by $1 \%$ of the wages, the average increase of life expectancy is 0.008204 . Thereby, it can be observed that the $c(1)$ and $c(2)$ coefficients are significantly different from zero, at the sample level, as well as for the entire population. The probability that the null hypothesis is true is smaller than $5 \%(p=0.0000$ for both coefficients), so it can be stated that the null hypothesis is rejected and the only accepted true hypothesis is the alternative $\mathrm{H}_{1}(\mathrm{c}(1) \neq 0$, and $\mathrm{c}(2) \neq 0)$.

In order to measure the intensity of the endogenous variable's dependency from regression factors, the determination coefficient needs to be found out. Based on the obtained results, at a sample level, there is a medium link between the endogenous and exogenous variables, the $R^{2}$ adjusted is equal to 0.595698 . In order to study the size of $R^{2}$ adjusted for the entire population, we used the Fisher test.

Because $F_{\text {calc }}=36.36158>F_{\text {tab }}=4.24$ (Andrei, et. al, 2008) the results prove that the null hypothesis is rejected, so the influence of exogenous variables is significant. If the difference between $F_{\text {calc }}$ and $F_{\text {tab }}$ increases, the link between the two variables is medium at the level of the entire population.

In order to find out the autocorrelation of errors, we have used the Durbin-Watson test (Andrei et. al., 2008: 126). Working with a significance level of $\alpha=0.05$, the number of exogenous variables is $k=1$ and the number of observations is $T=25$, from the Durbin-Watson distribution table we find the following values: $d_{1}=1.28$ and $d_{2}=1.45$. Because $0<D W_{\text {calc }}=0.147086<d_{1}=1.28$, the errors are auto correlated in a positive manner, the $\mathrm{H}_{0}$ hypothesis is rejected, so the independence hypothesis of errors is not verified. 
In order to eliminate the autocorrelation phenomenon, we used the Cochrane-Orcutt method (Stancu, 2011: 246), which states that estimating the $\rho$ coefficient and making a regression through quasi-difference in a model that has the form:

$$
\begin{aligned}
& Y_{t}=c(1) X_{1 t}+c(2)+\varepsilon_{t} \\
& Y_{t-1}=c(1) X_{1 t-1}+c(2)+\varepsilon_{t-1}
\end{aligned}
$$

The $\rho$ coefficient is determined through direct regression of the residue on the delayed $(\mathrm{t}-1)$ value of it. Thus the quasi-differential value is written as:

$$
Y_{t}-\rho Y_{t-1}=c(1)\left(X_{1 t}-\rho X_{1 t-1}\right)+c(2)(1-\rho)+u_{t}
$$

Starting from the regression model that presents the relationship between life expectancy and wage, we re-estimated the value of the $\rho$ parameter and the previous regression, until the stability of the $c(1), c(2)$ parameters. The $\rho$ parameter is estimated using the smallest squares method, observing the hypothesis that the residues follow a self-regressive first order process: $\varepsilon_{\mathrm{t}}=\rho \varepsilon_{\mathrm{t}-1}+\mathrm{u}_{\mathrm{t}}$, where $\mathrm{u}_{\mathrm{t}}$ is a white noise and $\rho \in R$. After the calculus, we have obtained the value of $\rho$ as being equal to 0.93 .

Thus, based on the previous regression and the (4) base relation, corresponding to the $\rho$ parameter estimated above, we have equation:

$$
\log _{L} L E R_{t}-\rho L o g \_L E R_{t-1}=c(1)\left(\log \_W G R-\rho L o g \_W G R_{t-1}\right)+c(2)(1-\rho)+u_{t}
$$

We check the qualities of the new model, repeating the same stages as for the previous

\begin{tabular}{|c|c|c|}
\hline \multicolumn{3}{|c|}{$\begin{array}{l}\text { Method: Least Squares } \\
\text { Included observation: } 24 \text { after adjustments }\end{array}$} \\
\hline $\begin{array}{l}\text { Testing the significance of the } \\
\text { parameters (Student Test) }\end{array}$ & $\begin{array}{l}\mathrm{C}(1) \\
\mathrm{C}(2)\end{array}$ & $\begin{array}{l}0.018320(\text { Prob }=0.0109) \\
4.662447(\text { Prob }=0.0000)\end{array}$ \\
\hline Adjusted R-squared & & 0.226610 \\
\hline $\begin{array}{l}\text { Independence of errors } \\
\text { (Durbin Watson Test) }\end{array}$ & $\mathrm{DW}_{\text {calc }}$ & $1.697202^{*}$ \\
\hline $\begin{array}{l}\text { Homoscedasticity of errors } \\
\text { (White Test) }\end{array}$ & $\mathrm{F}_{\text {calc }}$ & $1.059619($ Prob=0.3644) \\
\hline $\begin{array}{l}\text { Normality of errors } \\
\text { (Jarque Bera Test) }\end{array}$ & $\mathrm{JB}_{\text {calc }}$ & $0.090778($ Prob $=0.955626)$ \\
\hline
\end{tabular}
model. In order to estimate its parameters, we used the generalized least squares method and we obtained the following results:

Table 2: Estimating the parameters of the new regression model (5)

* Working with a significance level of $\alpha=0.05$, the number of exogenous variables is $k=1$, and the number of observations is $T=24$, from the Durbin-Watson distribution table we find the following values: $\mathrm{d} 1=1.27$ and $\mathrm{d} 2=1.44$.

Source: authors' estimates using Eviews

The two parameters of the new model are significantly different from zero, at the sample level, but also at the level of the entire population, a fact that is confirmed by the probability that the null hypothesis is correct, a probability that is smaller than $5 \%$ in the case of the two parameters. 
In order to measure the intensity of the endogenous variable's dependency to regression factors, the determination coefficient is used. At a sample level, there is a medium to weak intensity link between the variables, because $R^{2}$ adjusted $=0.226610$. At the level of the entire population, we have used the Fisher test. Because $F_{\text {calc }}=7.739188>F_{\text {tab }}=4.24$ (Andrei, et. al, 2008), the result is that the null hypothesis is rejected (Prob $=0.010872$ ).

As to what the testing of the fundamental hypothesis referring to the random $u_{t}$ variable is concerned for the new model, we have reached the following conclusions:

-the independence hypothesis of the values of the residual variable $\mathrm{u}_{\mathrm{t}}$ is confirmed this time, because the Durbin-Watson statistic is equal to 1.697202 , so that $d_{2}=1.44<\mathrm{DW}_{\text {calc }}=$ $1.697202<4-\mathrm{d}_{2}=2.56$, meaning the errors of the model are independent;

-the homoscedasticity hypothesis of the residual variable $u_{t}$ is confirmed, because, as the data from table 2 shows, the probability related to the Fisher statistic is higher than $5 \%$, which determines the acceptance of the $\mathrm{H}_{0}$ hypothesis as being true.

-the normality hypothesis of the random variable $u_{t}$ is confirmed. One way of checking the normality of errors hypothesis is the Jarque-Berra test, which is an asymptotic test, usable in the case of a large volume sample, which follows a chi-squared distribution with two degrees of freedom (Mester, 2012: 150). Because the related probability of accepting the null hypothesis as being true (Prob=0.955626) is larger than $5 \%$, we can state that the normality of errors hypothesis cannot be rejected for the level of the entire population, the errors being normally distributed.

\subsection{The correlation between life expectancy and wage in Ukraine}

In terms of determining a link between the life expectancy $(Y)$ and the wages $(X)$ in Ukraine case, we will use also a simple linear regression model witch has the form:

$$
\log \_ \text {LEU }=c(1)^{*} \log _{-} W G U+c(2)+\varepsilon_{t}
$$

For determining the parameters of this regression, we use the generalized least squares method. Therefore, using the Eviews program we obtained the following results at a sample level:

Table 3: The estimation of regression equation (6)

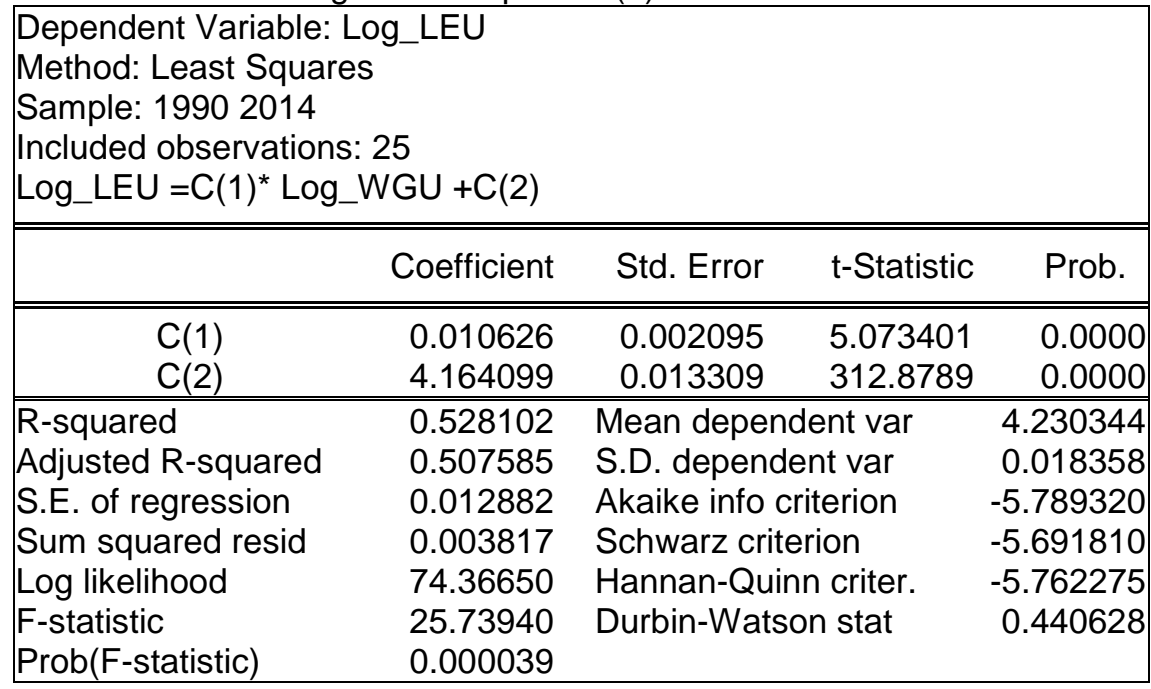

Source: authors' estimates using Eviews based on the data provided by World Bank and The State Statistics Service of Ukraine 
It can be seen that at a sample level, the function through which we define the model (6) is ascending. Therefore, we can notice the existence of a direct relation between wages and life expectancy in the Ukraine case. More than that, based on the data from the table above, we can see that for an increase by $1 \%$ of the wages, the average increase of life expectancy is 0.010626 . Following the calculations made with the help of the Eviews program, we can see that the two parameters are significantly different from zero at the sample level, as well as for the entire population. Because the probability that the null hypothesis is true is smaller than $5 \%$ ( $p=0.0000$ for both coefficients), it can be stated that the null hypothesis is rejected and the only accepted true hypothesis is the alternative $\mathrm{H}_{1}(\mathrm{c}(1) \neq 0$, and $\mathrm{c}(2) \neq 0)$.

As to what the $R^{2}$ adjusted determination coefficient is concerned, this is equal to 0.507585 at sample level, which suggests a medium link between the model variables. For the entire population, we used the Fisher test. Because $F_{\text {calc }}=25.73940>F_{\text {tab }}=4.24$ (Andrei, et. al, 2008) the results prove that the null hypothesis is rejected, so the influence of exogenous variables is significant and the link between the two variables is medium also at the entire population.

The Durbin-Watson test is used to find out the autocorrelation of errors (Andrei et al, 2008: 126). In order to choose a correct hypothesis, the $\mathrm{DW}_{\text {calc }}=0.44$ (table 3) statistic is determined. Working with a significance level of $\alpha=0.05$, the number of exogenous variables is $k=1$ and the number of observations is $T=25$, from the Durbin-Watson distribution table we find the following values: $d_{1}=1.28$ and $d_{2}=1.45$. Because $0<D W_{\text {calc }}=0.440628<d_{1}=1.28$, the errors are auto correlated in a positive manner and the $\mathrm{H}_{0}$ hypothesis is rejected, so the independence hypothesis of errors is not verified.

In order to eliminate the autocorrelation phenomenon, we used the Cochrane-Orcutt method (Stancu, 2011: 246) as in the case of Ukraine regression. After the calculus, we have obtained the value of $\rho$ as being equal to 0.77 and the equation is written:

$$
\log _{-} L E U_{t}-\rho \log \_L E U_{t-1}=c(1)\left(\log \_W G U-\rho L o g \_W G U_{t-1}\right)+c(2)(1-\rho)+u_{t}
$$

We check the qualities of the new model, repeating the same stages as for the previous model (6). For estimating the parameters of the new regression model (7), we used the generalized least squares method and we obtained the following results:

Table 4: Estimating the parameters of the new regression model (7)

\begin{tabular}{|c|c|c|}
\hline \multicolumn{3}{|c|}{$\begin{array}{l}\text { Dependent Variable: Log_LEU-0.77*Log_LEU }(-1) \\
\text { Method: Least Squares } \\
\text { Included observation: } 24 \text { after adjustments }\end{array}$} \\
\hline $\begin{array}{l}\text { Testing the significance of the } \\
\text { parameters (Student Test) }\end{array}$ & $\begin{array}{l}\mathrm{C}(1) \\
\mathrm{C}(2)\end{array}$ & $\begin{array}{l}0.007975(\text { Prob }=0.0426) \\
4.178692(\text { Prob }=0.0000)\end{array}$ \\
\hline Adjusted R-squared & & 0.136410 \\
\hline $\begin{array}{l}\text { Independence of errors } \\
\text { (Durbin Watson Test) }\end{array}$ & $\mathrm{DW}_{\text {calc }}$ & $1.203632^{*}$ \\
\hline $\begin{array}{l}\text { Homoscedasticity of errors } \\
\text { (White Test) }\end{array}$ & $F_{\text {calc }}$ & $0.711628($ Prob=0.5023) \\
\hline $\begin{array}{l}\text { Normality of errors } \\
\text { (Jarque Bera Test) }\end{array}$ & $\mathrm{JB}_{\text {calc }}$ & $0.384801($ Prob $=0.824976)$ \\
\hline
\end{tabular}

${ }^{*}$ Working with a significance level of $\alpha=0.01$, the number of exogenous variables is $k=1$, and the number of observations is $T=24$, from the Durbin-Watson distribution table we find the following values: $\mathrm{d} 1=1.037$ and $\mathrm{d} 2=1.199$.

Source: the authors' calculus 
The results related to the two parameters of the new model show that this two are significantly different from zero, at the sample level, but also at the level of the entire population, a fact that is confirmed by the probability that the null hypothesis is correct, a probability that is smaller than $5 \%$ in the case of the two parameters.

In order to measure the intensity of the link between the two variables, the determination coefficient is found. At a sample level, between the endogenous and exogenous variables is a medium to weak intensity link between the variables, because $R^{2}$ adjusted $=0.136410$. At the level of the entire population, we have used the Fisher test. Because $F_{\text {calc }}=4.632999>$ $\mathrm{F}_{\mathrm{tab}}=4.24$ (Andrei, et. al, 2008), the result is that the null hypothesis is rejected (Prob = $0.042594)$.

Testing the basic hypotheses referring to the random $u_{t}$ variable, we have reached the following conclusions:

-the independence hypothesis. Because the Durbin-Watson statistic is equal to 1.203632, the values of the residual variable $u_{t}$ is confirmed. So $d_{2}=1.199<D W_{\text {calc }}=1.203632<4-d_{2}=$ 2.801, meaning the errors of the model are independent for $\alpha=0.01$;

-the homoscedasticity hypothesis. In order to check this hypothesis, we use the White test. Because the probability related to the Fisher statistic is higher than $5 \%$, the homoscedasticity of the residual variable $u_{t}$ is confirmed which determines the acceptance of the $\mathrm{H}_{0}$ hypothesis as being true.

-the normality hypothesis of the random variable $u_{t}$ is confirmed. Because probability related to the Jarque Bera test (Prob=0.824976) is larger than $5 \%$, we can state that the normality of errors hypothesis cannot be rejected for the level of the entire population, the errors being normally distributed.

Also, it could be observed that the procedure for eliminating the autocorrelation of errors does not alter the structure of the regressions, both for Romania and the Ukraine. The $\mathrm{R}^{2}$ adjusted determination coefficient, as well as the $c(1)$ and $c(2)$ coefficients have values close to those from the first estimated equation (model (1) - the Romania case and model (6) Ukraine case).

\section{In conclusion}

Using the regression analysis, we have identified that between the life expectancy and the wages exists a direct correlation, both for Romania and the Ukraine. More than that, we find that in Romania, for an increase by $1 \%$ of the wages, the average increase of life expectancy is 0.008204 , while in Ukraine, for an increase by $1 \%$ of the wages, the average increase of life expectancy is 0.010626 . So, what we have assumed along with the theories presented within the first part of the work proved to be accurate, that both for Romania and Ukraine, between the wages and the life expectancy there is a direct connection. In other words, though the life expectancy bears the influence of GDP and the percentage allocated from GDP to the health system, the net income secured by each individual directly and consistently the life expectancy.

\section{References}

Andrei, T., Stancu, S., lacob, A.I. and Tusa, E. (2008). Introducere în econometrie utilizând EViews, Bucuresti: Economic Publishing House.

Cămăşoiu, C. (coord) (1994). Economia și sfidarea naturii, București: Editura Economică, Badulescu, D. and Badulescu, A. (2014). 'Medical Tourism: Between Entrepreneurship Opportunities and Bioethics Boundaries', Iranian Journal of Public Health, Vol. 43, Iss. 4, pp. 406-415.

Costea, C., Popescu, C. and Taşnadi, A. (2010). Criza e în noi, Bucuresti: Economic Publishing House. 
Cord, R.A. and Hammond, D. (2016). Milton Friedman. Contributions to Economics and Public Policy, New York: Oxford University Press.

Florea, A. (2008). Elemente de macroeconomie, Oradea: University of Oradea Publishing House.

Meșter, I. (2012). Econometrie, Oradea: University of Oradea Publishing House.

Pranab, B. and Udry, C. (2009). Development Microeconomics, New York: Oxford University Press.

Stancu, S. (2011). Econometrie. Teorie și aplicații utilizând Eviews, București: ASE Publishing House.

The National Institute of Statistics (2013). European Health \& Life Expectancy, EHLEIS National Report no.6, April, [online], available from: http://www.insse.ro/cms/files/pu-blicatii/Romania.pdf [11.07.2016].

The National Institute of Statistics of Romania (INSSE). Earnings - annual series from 1990 - 2014 [online], available from: http://www.insse.ro/cms/en/content/earnings-1938-annual-series-0 [10.06.2016].

The State Statistics Service of Ukraine. Income and Living Conditions [online], available from: http://www.ukrstat.gov.ua/ [10.06.2016].

The World Bank. Life expectancy at birth, total (years) - 1990-2014 [online], available from: http://data.worldbank.org/indicator/SP.DYN.LE00.IN [10.06.2016].

World Health Organization, Global Health Observatory data repository (category Mortality and global health estimates: Healthy life expectancy (HALE)) [online], available from: http://apps.who.int/gho/data/view.main.HALEXv?lang=en [11.07.2016].

\section{Bio-note}

Ramona Marinela Simut, Ph.D., is teaching assistant at University of Oradea, Faculty of Economics Sciences, Department of Economics. Her research interests include Statistics and Econometrics, European Economic Convergence, general aspects of Ecology and their impact of economy.

Perțicaș Diana Claudia, Ph.D., is lecturer at University of Oradea, Faculty of Economics Sciences, Department of Economics. Her main research interests include Ecology and Macroeconomics. The subject of her PhD research was "The role of renewable energies in sustainable economic development of Romania". 